PROCEEDINGS OF THE

AMERICAN MATHEMATICAL SOCIETY

Volume 137, Number 7, July 2009, Pages 2345-2350

S 0002-9939(09)09797-4

Article electronically published on January 30, 2009

\title{
VECTOR MEASURES AND THE STRONG OPERATOR TOPOLOGY
}

\author{
PAUL LEWIS, KIMBERLY MULLER, AND ANDY YINGST
}

(Communicated by N. Tomczak-Jaegermann)

\begin{abstract}
A fundamental result of Nigel Kalton is used to establish a result for operator valued measures which has improved versions of the Vitali-HahnSaks Theorem, Phillips's Lemma, the Orlicz-Pettis Theorem and other classical results as straightforward corollaries.
\end{abstract}

Each of $E, F, X, Y$, and $Z$ will denote a real $(\mathbf{R})$ Banach space. The space of all bounded linear functions (= operators) from $X$ to $Y$ will be denoted by $L(X, Y)$. Note that $X \cong L(\mathbf{R}, X)$. A generic $\sigma$-algebra of sets will be denoted by $\Sigma$. If $\left(A_{i}\right)_{i=1}^{\infty}$ is a sequence from $\Sigma$, then $\sigma\left(\left(A_{i}\right)_{i=1}^{\infty}\right)$ will denote the $\sigma$-algebra of subsets of $\bigcup_{i=1}^{\infty} A_{i}$ generated by $\left(A_{i}\right)_{i=1}^{\infty}$. If this sequence is pairwise disjoint, then $\sigma\left(\left(A_{i}\right)\right)$ is easily identifiable with $\mathcal{P}$, the power class of $\mathbf{N}$. If $\left(T_{i}\right)$ is a sequence in $L(X, Y)$, recall that $\left(T_{i}\right)$ converges to $T$ in the strong operator topology (sot) if and only if $\left(T_{i}(x)\right) \rightarrow T(x)$ in $Y$ for all $x \in X$. The reader should consult 3 and 2 for undefined terminology as well as the classical statements of the theorems and corollaries mentioned in this paper. The reader should note that there are non-separable subspaces of $\ell_{\infty}$ which do not contain $\ell_{\infty}$.

Theorem 1. Suppose that $\Sigma$ is a $\sigma$-algebra of subsets of $\Omega, E$ is separable, and $F$ is a Banach space which does not contain $\ell_{\infty}$ but does embed isomorphically into $\ell_{\infty}$. If $\mu: \Sigma \rightarrow L(E, F)$ is bounded and finitely additive and $\left(A_{i}\right)$ is a pairwise disjoint sequence from $\Sigma$, then there is a subsequence $\left(B_{i}\right)$ of $\left(A_{i}\right)$ so that $\mu: \sigma\left(\left(B_{i}\right)\right) \rightarrow$ $(L(E, F)$, sot $)$ is countably additive.

Proof. Suppose that $E, F, \Sigma,\left(A_{i}\right)$, and $\mu$ are as in the statement of the theorem. As before, let $\mathcal{P}$ be the power class of the positive integers, and identify $\mathcal{P}$ with $\sigma\left(\left(A_{i}\right)_{i=1}^{\infty}\right)$. If $b=\left(b_{i}\right) \in \ell_{\infty}$, let $T(b)=\int b d \mu$. Then $T: \ell_{\infty} \rightarrow L(E, F)$ is a bounded linear operator. For $x \in E$, define $T_{x}: \ell_{\infty} \rightarrow F$ by $T_{x}(b)=T(b)(x)$. Since $\ell_{\infty}$ does not embed in $F, T_{x}$ is weakly compact and therefore unconditionally converging; e.g., see [7, 6 6. p. 270].

Now let $T_{n}=T\left(e_{n}\right)$, where $\left(e_{n}\right)$ denotes the canonical unit vector basis of $c_{0} \subseteq$ $\ell_{\infty}$. Therefore $\sum T_{n}$ converges unconditionally in the strong operator topology. In

Received by the editors December 20, 2007, and, in revised form, September 16, 2008.

2000 Mathematics Subject Classification. Primary 28B05, 46B28, 46B25.

Key words and phrases. Operator valued measure, strong operator topology, Phillips's lemma, Orlicz-Pettis theorem.

(C)2009 American Mathematical Society 
fact, the map $\Delta: \ell_{\infty} \rightarrow L(E, F)$ defined by

$$
\Delta(\gamma) x=\sum_{i=1}^{\infty} \gamma_{i} T_{i}(x)=\sum_{i=1}^{\infty} \gamma_{i} T\left(e_{i}\right) x
$$

is bounded and linear. Let $L: F \rightarrow \ell_{\infty}$ be an isomorphism, and define operators $U: \ell_{\infty} \rightarrow L\left(E, \ell_{\infty}\right)$ and $V: \ell_{\infty} \rightarrow L\left(E, \ell_{\infty}\right)$ by

$$
U(b)(x)=L(T(b)(x))
$$

and

$$
V(b)(x)=\sum b_{i} L T_{i}(x)=L\left(\sum b_{i} T_{i}(x)\right) .
$$

Note that $U\left(e_{j}\right)(x)=L\left(T_{j}(x)\right)=V\left(e_{j}\right)(x)$ for each $j$. Proposition 5 of Kalton [6] applies and yields an infinite set $M \subseteq \mathbf{N}$ so that $U(t)=V(t)$ for all $t=\left(t_{i}\right) \in$ $\ell_{\infty}(M)$. Therefore $T(s)(x)=\sum s_{i} T_{i}(x)$ for $s=\left(s_{i}\right) \in \ell_{\infty}(M)$. The unconditional convergence of the series $\sum s_{i} T_{i}(x)$ and the preceding equality imply that

$$
\mu: \sigma\left(\left(A_{i}\right)_{i \in M}\right) \rightarrow(L(E, F), \text { sot })
$$

is countably additive.

In Corollary 5 below, we show that Theorem 1 leads to an improvement of Phillips's Lemma. Among the many consequences of Phillips's Lemma is a quick proof that $c_{0}$ is not complemented in $\ell_{\infty}$. The next result shows that this complementation result follows immediately from Theorem 1.

Corollary 2. $c_{0}$ is not complemented in $\ell_{\infty}$.

Proof. Suppose that $P: \ell_{\infty} \rightarrow c_{0}$ is a projection, and let $\mu: \mathcal{P} \rightarrow c_{0} \cong L\left(\mathbf{R}, c_{0}\right)$ be the bounded and finitely additive measure so generated. By Theorem 1, there is a subsequence $\left(n_{i}\right)$ of the positive integers so that $\mu: \sigma\left(\left(n_{i}\right)\right) \rightarrow L\left(\mathbf{R}, c_{0}\right)$ is countably additive in the strong operator topology ( $=$ the norm topology in this case). Clearly this is false since $\left\|\mu\left(n_{i}\right)\right\|=1$ for all $i$.

Now suppose that $\Sigma$ is a $\sigma$-algebra of subsets of $\Omega$, and $\lambda: \Sigma \rightarrow \mathbf{R}^{+}$is an extended $\mathbf{R}$-valued and countably additive set function. Let $X$ be a Banach space and $\mu_{n}: \Sigma \rightarrow X$ be countably additive so that $\mu_{n} \ll \lambda$ for all $n$. Suppose that $\left(\mu_{n}(A)\right)$ is norm convergent in $X$ for all $A \in \Sigma, \epsilon>0, \lambda\left(A_{n}\right) \rightarrow 0$, and $\left\|\mu_{n}\left(A_{n}\right)\right\|>2 \epsilon$ for all $n$. By a standard Cantor diagonalization argument, one may choose a subsequence $\left(A_{n_{i}}\right)$ of $\left(A_{n}\right)$ so that

$$
\left\|\mu_{n_{i}}\left(A_{n_{i}} \backslash \bigcup_{j>i} A_{n_{j}}\right)\right\|>\epsilon
$$

for each $i$.

Let $\nu_{i}=\mu_{n_{i}}, B_{i}=A_{n_{i}} \backslash \bigcup_{j>i} A_{n_{j}}, U=\left\{\nu_{i}\left(B_{j}\right): i \geq 1, j \geq 1\right\}$, and $Y=[U]=$ $\overline{\operatorname{span}}(U)$. Then $Y$ is separable. Thus the space $c(Y)=\left\{\left(y_{n}\right): n \in \mathbf{N}\right\} \subseteq Y^{\mathbf{N}}$ of all convergent sequences in $Y$ is separable when endowed with the usual pointwise operations and the sup norm. Define $\tau: \sigma\left(\left(B_{i}\right)\right) \rightarrow c(Y)$ by

$$
\tau(A)=\left(\nu_{i}(A)\right), A \in \sigma\left(\left(B_{i}\right)\right) .
$$

Note that $c(Y) \cong(L(\mathbf{R}, Y)$, sot $)$. By Theorem 1 , we know that there is a subsequence $\left(B_{i_{j}}\right)$ so that $\tau: \sigma\left(\left(B_{i_{j}}\right)\right) \rightarrow(L(\mathbf{R}, c(Y))$, sot $)$ is countably additive. However, this is impossible since $\left\|\nu_{i}\left(B_{i}\right)\right\|>\epsilon$ for each $i$. Therefore Theorem 1 produces the following improved versions of the Vitali-Hahn-Saks Theorem [4, p. 24], and [5. p. 158]. 
Corollary 3. (i) If $\epsilon>0, \lambda: \Sigma \rightarrow \mathbf{R}^{+}$is extended $\mathbf{R}$-valued and countably additive, $\left(\mu_{n}\right)$ is a sequence in ca $(\sigma, X)$ with $\mu_{n} \ll \lambda$ for each $n$, and $\left(A_{n}\right)$ is a pairwise disjoint sequence from $\Sigma$ so that $\left(\lambda\left(A_{n}\right)\right) \rightarrow 0$ and $\left\|\mu_{n}\left(A_{n}\right)\right\|>\epsilon$ for all $n$, then no infinite subsequence of $\left(\mu_{n}\right)$ can converge setwise on all elements of $\left(A_{n}\right)$.

(ii) If $\left(\mu_{n}\right)$ is a sequence in ca $(\Sigma), \epsilon>0$, and $\left(A_{n}\right)$ is a pairwise disjoint sequence in $\Sigma$ so that $\left|\mu_{n}\left(A_{n}\right)\right|>\epsilon$ for each $n$, then no infinite subsequence of $\left(\mu_{n}\right)$ can converge setwise on all elements of the sequence $\left(A_{n}\right)$.

Recall that a finitely additive set function $\mu$ defined on an algebra $\mathcal{A}$ is said to be strongly additive provided that $\mu\left(A_{i}\right) \rightarrow 0$ whenever $\left(A_{i}\right)$ is a pairwise disjoint sequence from $\mathcal{A}$. It is easy to see that a family of countably additive set functions defined on a $\sigma$-algebra $\Sigma$ is uniformly countably additive if and only if it is uniformly strongly additive. We say that $\mu: \mathcal{A} \rightarrow X$ is weakly strongly additive provided that $x^{*} \circ \mu: \mathcal{A} \rightarrow \mathbf{R}$ is strongly additive for all $x^{*} \in X^{*}$. Certainly every strongly additive set function is weakly strongly additive. If $c_{0} \hookrightarrow X \hookrightarrow L\left(X^{*}, \mathbf{R}\right) \cong X^{* *}$, then the following proposition makes it clear that there are weakly strongly additive set functions which are not strongly additive.

Proposition 4. Every weakly strongly additive set function $\mu: \mathcal{A} \rightarrow X$ is strongly additive iff $c_{0} \nrightarrow X$.

Proof. Suppose that $c_{0} \hookrightarrow X$ and let $\mathcal{A}$ be the finite-cofinite algebra of subsets of N. Define $\mu: \mathcal{A} \rightarrow X$ by

$$
\mu(A)=\sum_{n \in A} e_{n}, A \text { finite }
$$

and

$$
\mu(A)=\sum_{n \in A^{c}} e_{n}, A \text { cofinite. }
$$

Suppose that $\left(B_{i}\right)$ is a pairwise disjoint sequence from $\mathcal{A}$. Note that there is an $N$ so that if $n>N$, then $B_{n}$ is finite. Let $x^{*} \in X^{*}$, and choose $\lambda=\left(\lambda_{i}\right) \in \ell_{1}$ so that $x^{*} \mu(A)=\langle\lambda, \mu(A)\rangle$ for each $A$. Since $\sum_{i>n}\left|\lambda_{i}\right| \rightarrow 0$ as $n \rightarrow \infty$ and at most one member of the sequence $\left(B_{i}\right)$ is cofinite, it follows that $\left(x^{*} \mu\left(B_{n}\right)\right) \stackrel{n}{\rightarrow} 0$. Thus $\mu$ is weakly strongly additive and not strongly additive.

Now suppose that $\mathcal{A}$ is an algebra of sets and $\mu: \mathcal{A} \rightarrow X$ is weakly strongly additive and not strongly additive. Let $\left(A_{i}\right)$ be a pairwise disjoint sequence in $\mathcal{A}$ and $\epsilon$ be a positive number so that $\left\|\mu\left(A_{i}\right)\right\|>\epsilon$ for each $i$. If $x^{*} \in X^{*}$, then the weak strong additivity guarantees that $\sum\left|x^{*} \mu\left(A_{i}\right)\right|<\infty$. Thus $\sum \mu\left(A_{i}\right)$ is weakly unconditionally convergent and not unconditionally convergent. Classical results of Bessaga and Pelczynski [1] ensure that $c_{0} \hookrightarrow X$.

Let $c_{0}(Y)$ be the subspace of $c(Y)$ consisting of null sequences.

Corollary 5 (Phillips's Lemma). If $E$ and $F$ are separable, $\mu_{n}: \mathcal{P} \rightarrow L(E, F)$ is strongly additive in the sot for each $n$, and $\left(\mu_{n}(A)\right) \rightarrow 0$ in the sot for each $A$, then

$$
\sum_{k \in A} \mu_{n}(k) \stackrel{n}{\rightarrow} 0
$$

in the sot uniformly for $A \in \mathcal{P}$.

Proof. Suppose not, and select $\epsilon>0,\left(A_{i}\right)$ from $\mathcal{P}, x \in E$, and a subsequence $\left(\lambda_{i}\right)$ of $\left(\mu_{n}\right)$ so that $\left\|\sum_{k \in A_{i}} \lambda_{i}(k) x\right\|>2 \epsilon$ for each $i$. Using the unconditional convergence 
of $\sum \lambda_{i}(k) x$ (strong additivity in the sot) and the fact that $\left(\mu_{n}(A) x\right) \rightarrow 0$ for all $A$, obtain a strictly increasing sequence $\left(N_{i}\right)$ of positive integers so that

$$
\left\|\sum_{k \in A_{n_{i}} \cup\left[N_{i-1}, N_{i}\right)} \lambda_{N_{i}}(k) x\right\|>\epsilon
$$

for each $i$. Let $B_{i}=A_{N_{i}} \cap\left[N_{i-1}, N_{i}\right)$, let $\Sigma=\sigma\left(\left(B_{i}\right)\right)$, and set $\nu(A)(u)=$ $\left(\lambda_{i}(A)(u)\right)_{i} \in c_{0}(F)$ for $A \in \Sigma$ and $u \in E$. Inequality $(*)$ and an application of Theorem 1 to the set functions $\nu$ just defined are incompatible.

In the next corollary, $b f a(\mathcal{P}, X)$ is the set of all bounded and finitely additive set functions $\mu: \mathcal{P} \rightarrow X$. Equip $b f a(\mathcal{P}, X)$ with the sup norm; i.e., $\|\mu\|=$ $\sup \{\|\mu(A)\|: A \in \mathcal{P}\}$.

Corollary 6. If $X^{*}$ is separable and $\left(\mu_{n}\right) \rightarrow 0$ weakly in bfa $(\mathcal{P}, X)$, then

$$
\sum_{k=1}^{\infty}\left|x^{*}\left(\mu_{n}(k)\right)\right| \stackrel{n}{\rightarrow} 0
$$

for all $x^{*} \in X^{*}$.

Proof. Since $x^{*} \mu_{n}$ is bounded and finitely additive for all $x^{*} \in X^{*}, x^{*} \mu_{n}$ is strongly additive. Further, $\left(x^{*} \mu_{n}(A)\right) \stackrel{n}{\rightarrow} 0$ for all $x^{*} \in X^{*}$ and $A \in \mathcal{P}$. Therefore $\left(\mu_{n}(A)\right) \rightarrow 0$ in the $(s o t)$ when $X$ is canonically embedded in $X^{* *}=L\left(X^{*}, \mathbf{R}\right)$. By the preceding corollary, $\sum_{k \in A} x^{*} \mu_{n}(k) \stackrel{n}{\rightarrow} 0$ uniformly for $A \in \mathcal{P}$. Thus $\sum_{n=1}^{\infty} \mid x^{*}\left(\mu_{n}(k) \mid \stackrel{n}{\rightarrow} 0\right.$.

Since the sup norm on $c a(\mathcal{P})$ is equivalent to the total variation norm when $\ell_{1}$ is interpreted in this space, Corollary 6 shows that $\ell_{1}$ has the Schur property.

Corollary 7 (Vitali-Hahn-Saks-Nikodym Theorem). If $X$ and $Y$ are separable, $\mu_{n}: \Sigma \rightarrow L(X, Y)$ is a bounded and finitely additive set function which is strongly additive in the sot for each $n$, and $\left(\mu_{n}(A)\right) \rightarrow 0$ in the sot for each $A$, then $\sup _{n}\left\|\mu_{n}\left(A_{i}\right) x\right\| \stackrel{i}{\rightarrow} 0$ for each $x \in X$ whenever $\left(A_{i}\right)$ is pairwise disjoint in $\Sigma$.

Proof. Suppose not. Let $\epsilon>0,\left(A_{i}\right)$ be a pairwise disjoint sequence in $\Sigma$, and $x \in X$ so that $\sup _{n}\left\|\mu_{n}\left(A_{i}\right) x\right\|>\epsilon$ for each $i$. Let $\Sigma_{1}=\sigma\left(\left(A_{i}\right)\right)$, and define $\nu: \Sigma_{1} \rightarrow$ $L\left(X, c_{0}(Y)\right)$ by $\nu(A)(u)=\left(\mu_{n}(A) u\right)_{n=1}^{\infty}$. The preceding inequality and Theorem 1 applied to the vector measure $\nu$ provide the contradiction which completes the proof.

The proof of the Vitali-Hahn-Saks-Nikodym Theorem in Chapter 1 of [4 shows that there is no loss of generality in insisting that $Y$ be separable in the preceding corollary.

Corollary 8. Suppose that $\mu: \mathcal{P} \rightarrow X$ is bounded and finitely additive, $\Gamma$ is a subset of $X^{*}$ which separates the points of $X$, and $\mu$ is countably additive in the weak topology on $X$ defined by $\Gamma$. The set function $\mu$ is countably additive in the norm topology if and only if $U=\overline{c o}\{\mu(A): A \in \mathcal{P}, A$ finite $\}$ is $\Gamma$-closed.

Proof. Since $\Gamma$ separates the points of $X$ and $\mu$ is $\Gamma$-countably additive, $\mu$ is countably additive in the norm topology if $\mu$ is strongly additive with respect to the norm on $X$. Suppose then that $U$ is $\Gamma$-closed, and let $W=[U]$. The $\Gamma$-countable additivity of $\mu$ ensures that $\mu(\mathcal{P}) \subseteq W=L(\mathbf{R}, W)$. By Theorem 1 , there does not 
exist $\epsilon>0$ and a disjoint sequence $\left(A_{i}\right)$ in $\mathcal{P}$ so that $\left\|\mu\left(A_{i}\right)\right\|>\epsilon$ for all $i$; i.e., $\mu$ is strongly additive.

Conversely, suppose that $\mu: \mathcal{P} \rightarrow(X,\|\bullet\|)$ is countably additive. Thus $\mu$ has relatively weakly compact range, and $U$ is weakly compact. Then $U$ is $\Gamma$-compact and $\Gamma$-closed since $\Gamma$ separates points.

Since a norm-closed convex set is weakly closed, the preceding corollary certainly demonstrates that a weakly countably additive vector measure on a $\sigma$-algebra is countably additive. Thus this corollary contains the measure-theoretic version of the Orlicz-Pettis Theorem.

Next we note that Theorem 1 also yields a quick proof of the standard statement of the Orlicz-Pettis Theorem: If $\sum x_{n}$ is weakly subseries convergent in $X$, then $\sum x_{n}$ is unconditionally convergent in $X$.

Note first that $\sum_{k=1}^{\infty}\left|x^{*}\left(x_{n}\right)\right|<\infty$ for all $x^{*} \in X^{*}$, and let $Y=\left[\left\{x_{n}: n \in \mathbf{N}\right\}\right]$. Suppose that $\sum x_{n}$ is not unconditionally convergent. Let $\epsilon>0, \sigma: \mathbf{N} \rightarrow \mathbf{N}$ be a permutation, and $\left(p_{i}\right)$ and $\left(q_{i}\right)$ be intertwining sequences of natural numbers so that $\left\|w_{i}\right\|>\epsilon$ for all $i$, where $w_{i}=\sum_{n=p_{i}}^{q_{i}} x_{\sigma(n)}$. The hypothesis and the weak absolute summability of $\sum x_{n}$ noted above guarantee that $\nu(S)=$ weak-lim $\sum_{i \in S} w_{i}$ defines a bounded and finitely additive set function $\nu: \mathcal{P} \rightarrow Y$. Apply Theorem 1 to obtain a contradiction.

Corollary 9. If $K$ is a relatively weakly compact subset of $c a(\Sigma)$, then the elements of $K$ are uniformly countably additive.

Proof. As noted above, it suffices to show that $K$ is uniformly strongly additive. Let $\left(\mu_{n}\right)$ be a sequence from $K$, and, without loss of generality (Eberlein-Smulian Theorem), suppose that $\left(\mu_{n}\right)$ converges weakly to a point in $c a(\Sigma)$. Let

$$
\nu(A)=\left(\mu_{n}(A)\right)_{n=1}^{\infty}
$$

for $A \in \Sigma$, and let $c$ denote the space of all convergent sequences of real numbers equipped with the sup norm. Then $\nu: \Sigma \rightarrow L(\mathbf{R}, c)$ satisfies the hypotheses of Theorem 1 , and we conclude that $\left(\mu_{n}\right)$ is uniformly strongly additive.

At a crucial point in the proof of Theorem 1 above we used the following theorem of Rosenthal [7]: If $\ell_{\infty}$ does not embed in $F$, then every operator $T: \ell_{\infty} \rightarrow F$ is weakly compact. A key ingredient in the proof of Rosenthal's theorem is the following lemma.

Rosenthal's Lemma. If $\mathcal{A}$ is an algebra of sets, $\left(\mu_{n}\right)$ is a uniformly bounded sequence of non-negative and real valued measures on $\mathcal{A},\left(A_{n}\right)$ is a pairwise disjoint sequence of sets in $\mathcal{A}$, and $\epsilon>0$, then there is a subsequence $\left(n_{k}\right)$ of positive integers so that $\sum_{i=1, i \neq j}^{N} \mu_{n_{j}}\left(A_{n_{i}}\right)<\epsilon$ for each $N$ and for each $j$.

One may check Chapter 1 of [4 for numerous applications of Rosenthal's Lemma. We close with a particularly quick inductive proof of this lemma. The argument below naturally generates a subsequence $\left(\nu_{k}\right)$ of $\left(\mu_{n}\right)$ so that the co-finite and terminal subsequences of $\left(\nu_{k}\right)$ automatically satisfy the conclusion of Rosenthal's Lemma. 
Lemma 10. Suppose that $\left(\mu_{n}, A_{n}\right)$ is a sequence of pairs of measures and sets so that $\left(A_{n}\right)$ is distinct and pairwise disjoint and $\left(\mu_{n}\right)$ is uniformly bounded. If $\epsilon>0$, then there is an $n_{0}$ so that $\left\{n: \mu_{n}\left(A_{n_{0}}\right)<\epsilon\right\}$ is infinite.

Proof. Suppose that $\left\|\mu_{n}\right\|<M$ for each $n$, and choose $N$ so that $N \epsilon>M$. Choose distinct positive integers $i_{1}, i_{2}, \ldots, i_{N}$. If none of these choices satisfy the conclusion, then $S_{k}=\left\{n: \mu_{n}\left(A_{i_{k}}\right) \geq \epsilon\right\}$ is cofinite for $k=i_{1}, \ldots, i_{N}$. Thus $\bigcap_{k=1}^{N} S_{k}$ is cofinite. Consequently, $\left\{n: \mu_{n}\left(A_{i_{k}}\right) \geq \epsilon, k=1, \ldots, N\right\}$ is cofinite. Therefore, for some $n$, $\mu_{n}\left(\bigcup_{k=1}^{N} A_{i_{k}}\right) \geq N \epsilon$, and we have a contradiction.

Use the preceding lemma and choose $n_{1}$ so that $S_{1}=\left\{n>n_{1}: \mu_{n}\left(A_{n_{1}}\right)<\right.$ $1 / 2\}$ is infinite. Since $S_{1}$ is infinite and $\sum_{1=1}^{\infty} \mu_{n_{1}}\left(A_{i}\right)<\infty$, we may assume that $\sum_{i>n_{1}} \mu_{n_{1}}\left(A_{i}\right)<1 /\left(2^{2}\right)$. Now use the lemma again and choose $n_{2} \in S_{1}$ so that $S_{2}=\left\{n \in S_{1}: n>n_{2}, \mu_{n}\left(A_{n_{2}}<1 /\left(2^{2}\right)\right\}\right.$ is infinite. As above, we may assume that

$$
\sum_{i>n_{2}} \mu_{n_{2}}\left(A_{i}\right)<1 /\left(2^{3}\right)
$$

Continue this process inductively to manufacture a subsequence $\left(\nu_{k}\right)=\left(\mu_{n_{k}}\right)$. Note that if $\epsilon>0$ and $1 /\left(2^{k-1}\right)<\epsilon$, then $\left(\nu_{i}\right)_{i \geq k}$ satisfies the conclusion of Rosenthal's Lemma.

\section{REFERENCES}

[1] C. Bessaga and A. Pelczynski, On bases and unconditional convergence of series in Banach spaces, Studia Math. 17(1958), 151-164. MR0115069(22:5872)

[2] R. Bilyeu and P. Lewis, Uniform differentiability, uniform absolute continuity, and the VitaliHahn-Saks theorem, Rocky Mtn. J. Math. 10(1980), 533-557. MR590217 (82g:46083)

[3] J. Diestel, Sequences and Series in Banach Spaces, Grad. Texts in Math. 92, Springer, New York, 1984. MR737004 (85i:46020)

[4] J. Diestel and J.J. Uhl, Jr., Vector Measures, Amer. Math. Soc., Providence, RI, 1977. MR.0453964 (56:12216)

[5] N. Dunford and J. Schwartz, Linear Operators, Part I: General Theory, Wiley, New York, 1958. MR1009162 (90g:47001a)

[6] N. Kalton, Spaces of compact operators, Math. Ann. 208(1974), 267-278. MR0341154 $(49: 5904)$

[7] H. Rosenthal, On relatively disjoint families of measures, with some applications to Banach space theory, Studia Math. 37(1970), 13-36. MR0270122 (42:5015)

Department of Mathematics, University of North Texas, Box 311430, Denton, Texas $76203-1430$

Department of Mathematics and Computer Science, Lake Superior State University, 650 W. Easterday Avenue, Sault St. Marie, Michigan 49783-1699

Department of Mathematics, University of South Carolina, P.O. Box 889, Lancaster, South Carolina 29721 